\title{
Evidence-Base Rapid Review in Veterinary Medicine for Urgent and Emergent Decision-Making
}

\author{
Jeff M Perez* \\ Canadian Veterinary Hospital, Arab league Street and Jelaiah P.O. Box 209341, Qatar
}

Submission: January 29, 2019; Published: February 13, 2019

*Corresponding author: Jeff M Perez, Canadian Veterinary Hospital, Arab league Street and Jelaiah P.O. Box 209341, Doha, Qatar

\begin{abstract}
modern veterinary medicine has been increasingly focused on technical guidance, especially when rational results are necessary in animal's health and veterinarians seek to perform appropriate actions with specific objectives through logical steps using the best possible knowledge available. Thus, it becomes necessary to make a critical and proper evaluation of existing evidence. There is an increasing movement towards evidence-base narrative decision making for health systems and rapid reviews have emerged as an alternative. Rapid reviews are a form of evidence synthesis that may provide more timely information for decision making compared with standard systematic reviews.
\end{abstract}

Keywords: Rapid review; Evidence-base medicine; Systematic review; Animal's health

\section{Introduction}

The objective of modern veterinary medicine has been increasingly focused on technical guidance, especially when rational results are necessary in animal's health [1]. Otherwise, veterinarians seek to perform appropriate actions with specific objectives through logical steps using the best possible knowledge available $[2,3]$. Thus, it becomes necessary to make a critical and proper evaluation of existing evidence.

\section{Discussion}

The presentation of the evidence is established on multiple and very different sources in terms of strengths recommendations [4]. The more able way to show reliability have been the presentation of evidence-based medicine from systematic reviews and meta-analysis, allowing to make definitive and recommendable conclusions useful in clinical settings when they are used by health professionals [5]. Medical practice based on evidence-base medicine and technical guidance sustains a hierarchy of evidence $[6,7]$. The interpretation of this hierarchy has been based in last years on the study design of available studies, considering that some study designs offer better guarantees substantially reducing the bias [8]. Thus, a pyramid of many versions was proposed [9], but all of them with the same characteristic, showing the weaker design studies in the lower part being first the series of cases and case reports, followed by cases and controls and cohort studies. In the top, randomized controlled trials and finally with the best evidence, the systematic reviews and meta-analyzes at the tip [7].

This technical approach to the pyramid of evidence is correct in many case [10]. Nevertheless, in view of the inclusion of new approaches to the patient such as the health-related quality of life and scores of risks then, systematic reviews are limiting conclusions to only technical and quantitative results referring to population [11]. Leaving aside completely the subjective variables necessary for a human and kindness management of clinical practice. In fact, policymakers, health system managers and implementers are often faced with a strong barrier to the use of evidence synthesis to make decisions based on systematic reviews and meta-analysis [12].

The advantages of systematic reviews and meta-analysis are mainly the gathering, the evaluation and the synthesis of multiple studies [13]. In addition, the ability of meta-analysis to reduce biases and minimize random error is a critical aspect. Regarding the conclusions, systematic reviews and meta-analysis has been shown significance in the identification of the trends of the results with verifiable validity, generating more accurate information, more statistical power and with a better degree of evidence for clinical recommendations [14].

Thinking outside the box with systematic reviews and meta-analysis there are some limitations that hinders their use in every clinical situation specially in veterinary medicine, such as time consuming, the absence of sufficient published randomized trials to achieve inclusion criteria and in some situations the impractical results and the frequency of appearance of duplicate studies and repeated publications, followed by the problems with the rigor of the methodology and the quality of the investigations included in the analysis [15]. In addition, the incorrect combination of re- 
sults is frequent and most serious, the heterogeneity in the ways of measuring the variables of exposure and response and the heterogeneity among studies [16]. In fact, under these conditions the quality of results of systematic reviews and meta-analysis cannot be better than those obtained in an individual study with an adequate quality and sometimes become of little use for health policy and systems or marketing approval by regulatory entities [17].

In animal's health to the overall limitation adds the frequent incomplete search of literature, the variability in the evaluation of the quality of the articles, the difficulty to extrapolate results to the clinical practice, investigations with contexts, patients, measurements and different analyzes and a low knowledge of the subjects of study, but also in specific subject the absence of sufficient studies to create a meta-analysis, the absence of enough sample size in urgent and emergent decision making and the absence of organized work groups that guarantee long-term research conclusions $[18,19]$. There is an increasing movement towards evidence-base narrative decision making for health systems and rapid reviews have emerged as an alternative to address these obstacles above mentioned [20]. Rapid reviews can be viewed as a simplified approach to systematic reviews. Thus, follows most of the principle steps of a systematic review, using systematic and transparent methods to identify, select, critically appraise and analyze data from relevant research [21]. However, to provide timely evidence some of the components of a systematic review process are either simplified or omitted [22,23].

An evidence-base rapid review differs from a systematic review and meta-analysis because it is conducted for a short period of time and covers a limited number of resources [21]. A rapid review includes explicit strategies and a methodology with a rigorous structure of the evidence found. It is basically a pragmatic and useful alternative to systematic reviews and meta-analysis and basically, a rapid review has been developed in response to the demand for synthesis of evidence in an accelerated way to meet the needs of decision making in clinical practice. Although rapid reviews are created to have useful conclusions in clinical practice in a fast way, they must be scientifically rigorous [24]. One concern is that sometimes the quality of the evidence can be compromised, increasing the risk of losing evidence or inaccurate synthesis, essentially limiting the usefulness of the study [22]. Therefore, it is critical that a rapid review be rigorously and meticulously applied with transparent evidence synthesis methods [21]. To date, there are no studies comparing rapid systematic reviews and standard systematic reviews and standard definition for "evidence-base rapid review" does not yet exist and many methods and products have been described using this term [24].

Rapid reviews are a form of evidence synthesis that may provide more timely information for decision making compared with standard systematic reviews [21]. The methods of conducting rapid reviews varies widely and are typically done in less than 5 weeks [21]. Rapid Reviews are best designed for broader PICO questions (Population, Intervention, Control and Outcomes), new or emerging research topics, updates of previous reviews or guidelines, critical topics, to assess what is already known about a policy or practice using some systematic review methods $[5,23]$.

\section{Conclusion}

In conclusion, evidence-base rapid reviews could solve necessary answers with exceptional quality and consistently be able to give clear conclusions to clinical problems of interest for animal's health. However, it should be considered that they would not replace the systematic reviews and meta-analysis at all and are only useful when the necessary requirements cannot be met to carry out a systematic review and meta-analysis without compromising their quality and reliability. The objective of this mini-review is to give a perspective about pragmatic solution for the inability in veterinary medicine to follow in some subjects the minimal requirements to achieve systematic review and meta-analysis and more interesting to give a recommendation about the importance to include an evidence-base rapid review in each animal's health guidelines update in veterinary practice in the upcoming publications.

\section{Conflict of Interest}

The author has no conflict of interest to declare.

\section{References}

1. Woods A (2017) From One Medicine to Two: The Evolving Relationship between Human and Veterinary Medicine in England, 1791-1835. Bull Hist Med 91(3): 494-523.

2. Shurtz S, Fajt V, Heyns EP, Norton HF, Weingart S (2017) Teaching Evidence-Based Veterinary Medicine in the US and Canada. J Vet Med Educ 44(4): 660-668.

3. Reeve-Johnson L (2015) Evidence-based veterinary medicine. Vet Rec 177(15): 398.

4. Guyatt GH, Oxman AD, Vist GE, Kunz R, Falck-Ytter Y, et al. (2008) GRADE: an emerging consensus on rating quality of evidence and strength of recommendations. 336(7650): 924-926.

5. Rogers Van Katwyk S, Grimshaw JM, Mendelson M, Taljaard M, Hoffman SJ (2017) Government policy interventions to reduce human antimicrobial use: protocol for a systematic review and meta-analysis. Syst Rev 6(1): 256.

6. Atkins D, Best D, Briss PA, Eccles M, Falck-Ytter Y, et al. (2004) Grading quality of evidence and strength of recommendations. 328(7454): 1490.

7. Milano G (2015) The hierarchy of the evidence-based medicine pyramid: classification beyond ranking. 3(3): 101.

8. Shaneyfelt T (2016) Pyramids are guides not rules: the evolution of the evidence pyramid. Evid Based Med 21(4): 121-122.

9. Murad MH, Asi N, Alsawas M, Alahdab F (2016) New evidence pyramid. Evid Based Med 21(4): 125-127.

10. Rosner AL (2012) Evidence-based medicine: revisiting the pyramid of priorities. J Bodyw Mov Ther 16(1): 42-49.

11. Devji T, Johnston BC, Patrick DL, Bhandari M, Thabane L, et al. (2017) Presentation approaches for enhancing interpretability of patientreported outcomes (PROs) in meta-analysis: a protocol for a systematic survey of Cochrane reviews. BMJ Open 7(9): e017138. 
12. Tricco AC, Cardoso R, Thomas SM, Motiwala S, Sullivan S, et al. (2016) Barriers and facilitators to uptake of systematic reviews by policy makers and health care managers: a scoping review. Implement Sci 11:4.

13. Shamseer L, Moher D, Clarke M, Ghersi D, Liberati A, et al. (2015) Preferred reporting items for systematic review and meta-analysis protocols (PRISMA-P) 2015: elaboration and explanation. BMJ 350 g7647.

14. Moher D, Shamseer L, Clarke M, Ghersi D, Liberati A, et al. (2015) Preferred reporting items for systematic review and meta-analysis protocols (PRISMA-P) 2015 statement. Syst Rev 4: 1.

15. Weatherall M (2017) Systematic review and meta-analysis: tools for the information age. Postgrad Med J 93(1105): 696-703.

16. McKenzie JE, Beller EM, Forbes AB (2016) Introduction to systematic reviews and meta-analysis. Respirology 21(4): 626-637.

17. Beck CR, McKenzie BC, Hashim AB, Harris RC, Zanuzdana A, et al. (2011) Influenza vaccination for immunocompromised patients: systematic review and meta-analysis from a public health policy perspective. 6(12): e29249.

18. Sargeant JM, O'Connor AM (2014) Introduction to systematic reviews in animal agriculture and veterinary medicine. Zoonoses Public Health 61(1): 3-9.
19. Sargeant JM, O'Connor AM (2014) One-stop shopping for information on conducting systematic reviews and meta-analysis in animal agriculture and veterinary medicine. Zoonoses Public Health 61(1): 2.

20. Bender M (2014) The current evidence bases for the clinical nurse leader: a narrative review of the literature. J Prof Nurs 30(2): 110-123.

21. Garritty C, Stevens A, Gartlehner G, King V, Kamel C (2016) Cochrane Rapid Reviews Methods Group to play a leading role in guiding the production of informed high-quality, timely research evidence syntheses. Syst Rev 5(1): 184.

22. Hartling L, Guise JM, Kato E, Anderson J, Belinson S, et al. (2015) A taxonomy of rapid reviews links report types and methods to specific decision-making contexts. J Clin Epidemiol 68(12): 1451-1462e3.

23. Featherstone RM, Dryden DM, Foisy M, Guise JM, Mitchell MD, et al (2015) Advancing knowledge of rapid reviews: an analysis of results, conclusions and recommendations from published review articles examining rapid reviews. Syst Rev 4: 50.

24. Haby MM, Chapman E, Clark R, Barreto J, Reveiz L, et al. (2016) What are the best methodologies for rapid reviews of the research evidence for evidence-informed decision making in health policy and practice: a rapid review. Health Res Policy Syst 14(1): 83.

Your next submission with Juniper Publishers
will reach you the below assets
- Quality Editorial service
- Swift Peer Review
- Reprints availability
- E-prints Service
- Manuscript Podcast for convenient understanding
- Global attainment for your research
- Manuscript accessibility in different formats
( Pdf, E-pub, Full Text, Audio)
- Unceasing customer service
Track the below URL for one-step submission
https://juniperpublishers.com/online-submission.php

